\title{
Investigation of Low-Frequency Sound Colouration Treatments in Small Rooms by Means of Finite Element Analysis
}

\author{
Christos Sevastiadis \\ Electrical and Computer Engineering Department, Aristotle University of Thessaloniki, University Campus AUTH, \\ 54124, Thessaloniki, Greece \\ George Kalliris \\ Journalism and Mass Communication Department, Aristotle University of Thessaloniki, University Campus \\ AUTH, 54124, Thessaloniki, Greece
}

George Papanikolaou

Electrical and Computer Engineering Department, Aristotle University of Thessaloniki, University Campus AUTH, 54124, Thessaloniki, Greece

(Received 19 December 2008; revised 27 December 2009; accepted 19 April 2010)

Acoustics in small rooms suffer from resonances in low frequencies, resulting in the well-known sound colouration problem. In the present work, the finite element method was used to investigate specific case studies of proposed treatments of this problem. The treatments are based on techniques that make use of three basic mechanisms: wavelength, boundary impedance, and active control. The quality of the treatments is evaluated using two spatial statistical measures of the sound pressure level frequency responses regularly sampled in the listening area. The results demonstrate the advantage of the impedance- and active control mechanism-based techniques. Resonant panels and multiple source excitation treatments improve the sound field flatness.

\section{INTRODUCTION}

The acoustical behavior of small rooms is important to consider when they are used as performance studios, studio control rooms, audio program assessment rooms, measurement laboratories, small conference rooms, or even as simple dwellings. Their sizes are comparable to the low-frequency sound wavelengths, resulting in discrete resonances due to the produced standing waves. The uneven distributed nodes and antinodes of room modes are dominant in the sound pressure level (SPL) frequency response and spatial distribution. The modal behavior of the sound field reduces the quality of the perceived sound, causing the sound colouration effect ${ }^{1-3}$. This behavior also undermines the accurate prediction and the repeatability of acoustical and electroacoustical measurements in laboratories. The same problem applies to the transmission loss measurements in problems of sound insulation between rooms. ${ }^{4}$

There are two major issues that are concerned with lowfrequency sound colouration: study approach and treatment mechanisms. The low-frequency sound field in small rooms cannot be studied using the classical geometrical and statistical acoustics analysis because the sound field is not diffuse below the Schroeder frequency. Instead of the particle model approach, the fundamental wave theory analysis is more valid in low-frequency acoustics ${ }^{5,6}$. Regular geometric shapes, such as rectangles, cylinders, and prisms, can be investigated by applying analytical and semi-analytical solution methods in wave equations. The solution of the problem is sought in terms of the room's normal modes. By applying the normal mode expansion technique, both the frequency and transient responses of harmonic excitations can be obtained ${ }^{7}$. When the shape of the room is irregular the analytical methods become too difficult to be applied and numerical ones are used instead of them. The finite element method is a numerical approach that has been broadly used in acoustical problems ${ }^{8-13}$. While the modal expansion technique is complicated to use, the simple direct method is a convenient alternative to apply to the wave problem, thanks to today's ever increasing computer power and memory capabilities.

In general, there are three basic mechanisms that are encountered in related techniques. The first meachanism is aiming to redistribute the mode frequencies through wavelength control. Room size ratios are optimized by focusing on the mode's frequencies distribution statistics $1,1,14,15,17-20$. Splayed walls are applied, which decrease the probability of resonance degeneracy due to the uneven concentration of the mode frequency in the room. ${ }^{21}$ Scattering through obstacles and irregularities, especially through optimization techniques, induces more diffuse sound fields. The depth of the irregularities, however, needs to be large because of the low frequencies' long wavelengths. ${ }^{22-24}$

The second mechanism is the boundary impedance control. The applied techniques cause attenuation and perturbation of degeneracy resonances. Dissipation through light and heavy absorbing lining reduces the resonance peaks and decreases their decay time. This mechanism is the most frequently investigated field in the application of finite element analysis in room acoustics ${ }^{25-33}$. In practice, the thickness needed for absorption through porous layers has to be large because of the 